\title{
Analysis of Biliary Microbiota in Hepatobiliopancreatic Diseases Compared to Healthy People [MICROBILIO]: Study Protocol
}

\author{
Fernanda Sayuri do Nascimento \\ Universidade de Sao Paulo \\ Milena Oliveira Suzuki \\ Universidade de Sao Paulo \\ João Victor Taba \\ Universidade de Sao Paulo \\ Vitoria Carneiro de Mattos \\ Universidade de Sao Paulo \\ Leonardo Zumerkorn Pipek \\ Universidade de Sao Paulo \\ Eugênia Machado Carneiro D’Albuquerque \\ Hospital Santa Marcelina \\ Alberto Meyer ( $\square$ alberto.meyer@usp.br) \\ Universidade de São Paulo https://orcid.org/0000-0002-8408-0508 \\ Wellington Andraus \\ Universidade de Sao Paulo \\ João Renato Rebello Pinho \\ Universidade de Sao Paulo \\ Eduardo Guimarães Hourneaux de Moura \\ Universidade de Sao Paulo \\ João Carlos Setubal \\ Universidade de Sao Paulo \\ Luiz Augusto Carneiro-D'Albuquerque \\ Universidade de Sao Paulo
}

Research

Keywords: Pancreatic Diseases, Microbiota, Early Detection of Cancer, Dysbiosis, Biliary Tract Diseases

Posted Date: June 15th, 2020

DOI: https://doi.org/10.21203/rs.3.rs-34779/v1

License: () This work is licensed under a Creative Commons Attribution 4.0 International License. Read Full License

Version of Record: A version of this preprint was published at PLOS ONE on November 19th, 2020. See the published version at https://doi.org/10.1371/journal.pone.0242553. 


\section{Abstract}

Background: The performance of the microbiota is observed in several digestive tract diseases. Therefore, reaching the biliary microbiota may suggest ways for studies of biomarkers, diagnoses, tests and therapies in hepatobiliopancreatic diseases.

Methods: Bile samples will be collected in endoscopic retrograde cholangiopancreatography patients (case group) and living liver transplantation donors (control group). From the DNA extracted from the samples, the microbiomes will be sequenced by $16 \mathrm{~S}$ ribosomal RNA (rRNA) pyrosequencing methods. Student's t-test will be performed with SPSS version 20 for Windows and the Mann-Whitney test will be performed using R software and Python scripts. A principal component analysis signals will be developed as discriminative analysis.

Results: The primary expected results of the study is to characterize the specific composition of the biliary microbiota in situations of disease and health. In addition, it seeks to demonstrate the existence of changes in the case of illness and also possible disease biomarkers, diagnosis, interventions and therapies in hepatobiliopancreatic diseases.

Conclusions: We believe that the investigation of the biliary tract microbiota will provide benefits such as early diagnosis and new treatments. This should improve quality of life and survival, specially in malignant diseases. Thus, this research will give expectations to further investigation in the biliary microbiota.

Trial registration: NCT04391426. Registered 18 May 2020, https://clinicaltrials.gov/ct2/show/NCT04391426

\section{Introduction}

Microbioma is the set of microorganisms that occurs naturally in a particular site, such as the human gastrointestinal tract. Typically, it has trillions of microbes, including fungi, viruses and bacteria [1], which coexist with human cells.

Normally, the microbiome bacteria interact with the epithelial barrier, with immune cells modulating their response, in addition to influencing local metabolism through their own metabolites. This maintains homeostasis [2]. Thus, an imbalance of the microbiota, such as the use of antibiotics or due to bacterial translocation, can lead to the development of diseases. It does this through the proliferation of pathogenic bacteria, for example, which can greatly affect the host and have potential pathological implications. [2-4]

Studies have shown a close relation between dysbiosis and the outbreak of infections or chronic diseases. In 2019, Saus et al. [5] gathered data about the relation between the intestinal microbiota and the development of colorectal carcinoma (CRC), the most studied since the 1990s. Currently, it is known that in patients with this neoplasm there is a co-abundance of pro-inflammatory factors, opportunistic pathogens and other microbes. This is associated with metabolic dysfunction and the depletion of butyrate-producing bacteria, an important factor in intestinal homeostasis. With these studies, interest was raised in investigating other sites, such as the biliopancreatic tract (BPT). In 2015, Mitsuhashi et al. showed the association of the oral microbiota with the pancreatic carcinogenesis process. In periodontal diseases, Fusobacterium can be translocated via lymphohematogenous pathways, leading to pancreatic dysbiosis. This would be associated with malignancy in the progression of pancreatic adenocarcinoma and worse prognosis. [4]

Thus, research was conducted in rats, finding an association between the components of the tumour microbiota and the speed of progression of biliopancreatic disease [6]. In pancreases of rats and humans with pancreatic duct adenocarcinoma (PDAC), a greater abundance of Malassezia spp. was found compared to bowel or pancreas controls without the disease. Due to the presence of the fungus, there is greater activation of mannosebinding lectin (MBL) and, consequently, the complement cascade is activated, leading to greater inflammation in the pancreas, which accelerates tumour progression $[6,7]$.

Traditionally, BPT neoplasia are diagnosed at an advanced stage, despite the improvement in the quality of diagnostic imaging. For early diagnosis, Mendez et al. conducted an experiment with PDAC-mutated mice before they developed the disease. By DNA sequencing of the fecal microbiota bacteria, it was found that, with the progression of pancreatic carcinogenesis, there was a change in the bacterial composition. The metabolites of these bacteria associated with the tumour promote greater production of polyamines, which increases as the neoplasia develops. Then, the dosage of polyamines could be used as a biomarker to track the progression of adenocarcinoma [8]. The analysis of the fecal microbiota, therefore, would be a possibility for early diagnosis of PDAC, prompting research in human patients at high risk for carcinogenesis.

Among the therapies, the most commonly used treatments for neoplasia are chemotherapy and radiotherapy. In BPT carcinomas, however, these methods have low sensitivity [9], which can be attributed, according to studies, to tumor dysbiosis. As previously mentioned, unchanged symbiotic microbiota mediates the immune response. Thus its imbalance decreases the expression of genes related to inflammation, phagocytosis, antigen presentation and adaptive immune response. On the other hand, genes related to tissue development, cancer and metabolism are stimulated [10]. Thus, the use of chemotherapeutic drugs loses effectiveness due to this negative regulation of the immune system's anti-tumor capacity. An example of this is oxaliplatin, whose effect is to stimulate the production of reactive oxygen species to promote DNA damage and tumor cell apoptosis. In mice injected with colon carcinoma cells, their cytotoxic effect decreased after being treated with antibiotics. $[9,10]$ 
There have been several advances in the introduction of new chemical compounds that interfere in specific signalling pathways of carcinogenesis, also affected by the microbiome. Because of this association, microbial agents and their metabolites are being tested to develop treatments that can reduce the tumor and are potentially preventive [11]. This was observed in studies with species of Lactobacillus, which modulates the expression of some enzymes such as beta-glucuronidase. The action of the enzyme is reduced by bacilli. This acts in the disjunction of carcinogenic agents, converting pro-carcinogens into their active form [12]. Lenoir et al. also demonstrated that L.casei has anti-tumor properties by decreasing the T-reg response and increasing Th17, promoting a decrease in CRC in rats. Thus, the microbe proved to be a protector and a new therapeutic alternative to carcinoma. $[11,13]$

Even with current treatments, mortality in some groups of BPT malignancies remains high, with low survival rates. However, in recent studies in patients with pancreatic duct adenocarcinoma, it was found that the greater variety of the tumor microbiota and the predominance of specific bacterial genera are related to a longer survival time when treated surgically [6]. Considering this recent progress, improvement is expected for the coming decades.

Thus, the performance of the microbiota is observed in all clinical and pathological stages of carcinogenesis, from its development, diagnosis and treatment, including prognosis and survival. However, there is a lack of studies on biliary microbiota and its relation with hepatobiliopancreatic diseases. Therefore, further investigation is necessary, since researching the biliary microbiota may suggest ways for studies of biomarkers, diagnoses, interventions and therapies in hepatobiliopancreatic diseases.

\section{Aim}

In this study, our aim will be to characterize the specific composition of the biliary microbiota in patients with hepatobiliopancreatic diseases compared to healthy controls, using $16 \mathrm{~S}$ ribosomal RNA (rRNA) pyrosequencing methods.

\section{Material And Methods}

\section{- Study design and patients}

This is a case-control study that will be carried out at the Department of Gastroenterology of Clinicas Hospital in the Faculty of Medicine of the University of São Paulo.

Patients who will undergo endoscopic retrograde cholangiopancreatography (ERCP) and donors previously selected to interventional liver transplantation will be recruited for the collection of bile, configuring case and control groups, respectively. The project outline is illustrated in Fig. 1.

\section{- External Validation}

All patients who will not be included, with a condition for which a procedure is planned, such as ERCP or hepatectomy for liver transplantation, will have their records noted and stored, including information on date, sex, age, Body Mass Index (BMI) and the reason for non-inclusion or exclusion.

\section{- Patients who will undergo ERCP (case group)}

Inclusion criteria

- Patients over 18 years old

- Patients previously scheduled for ERCP

- Cannulation of the bile duct, via the transpapillary route, with the aid of a papillotome with an end kept sterile until contact with the papilla

Exclusion Criteria

- Use of antibiotics during ERCP or in the last 2 months prior to the procedure

- Emergency ERCP

- Pregnancy

- Uncorrected coagulopathy

History of previous ERCP will not be considered an exclusion criterio.

- ERCP Technical Description

The procedure will be performed under conscious sedation or general anesthesia, at the discretion of the medical team responsible for carrying out the procedure. 
Before the beginning of ERCP, the end of the papillotome will be covered with sterile surgical plastic (plastic cover for videolaparoscopy), in order to avoid its contamination during passage through the working channel of the duodenoscope and contact with the digestive tract, up to the greater duodenal papilla.

The duodenoscope will be introduced according to the usual technique and positioned in front of the greater duodenal papilla. The papillotome with a sterile end will then be passed through the working channel of the duodenoscope. Before attempting cannulation of the papilla, air will be injected through the papillotome injection channel to remove the sterile plastic from its end.

When performing cannulation of the bile duct, with the aid of endoscopic and radioscopic vision, the route of the papillotome injection until bile return will be aspirated with a sterile $5 \mathrm{ml}$ syringe, to confirm the correct positioning of the instruments in the bile duct. Then, $1 \mathrm{ml}$ of bile will be aspirated, which will be sent for analysis of the microbiota.

If there is contamination of the papillotome prior to contact with the papilla, the patient will be excluded from the protocol.

\section{- Living liver transplantation donors (control group)}

The correct selection of donors for living donor liver transplantation is essential not only to decrease the risk of complications for donors, but also to increase graft and recipient survival.

First, there must be ABO blood type compatibility. Then, the compatibility between the weight and height of the patient and the donor is analysed. Subsequently, the size of the liver to be donated is evaluated, and it is necessary to calculate the relation between the weight of the liver (donor) with the recipient. Finally, the entire anatomy of the donor and recipient is evaluated, such as veins, arteries and bile ducts.

Inclusion Criteria

- Patients over 18 years old up to 55 years old

- Previously selected patients with scheduled surgery

- BMl: $18 \mathrm{~kg} / \mathrm{m}^{2}$ to $28 \mathrm{~kg} / \mathrm{m}^{2}$

- Blood typing identical to the recipient

- Absence of significant medical, psychiatric problems or previous abdominal surgery

- - Normal laboratory tests: liver function tests, blood count, coagulogram, pregnancy test and serology for hepatitis B, C and HIV

- Normal imaging exams: CT of the abdomen and pelvis with liver volume (remaining volume $-30-40 \%$ of the total liver volume), MRI with cholangioresonance

Exclusion Criteria

- Use of antibiotics in the last 2 months prior to the procedure

- Pregnancy

- Uncorrected coagulopathy

- Description of the interventional liver transplantation technique

The hepatectomy procedure for related living donor liver transplantation, left lobe or right lobe will be performed under general anesthesia.

Started by a Makuuchi incision ("J" incision), followed by positioning the retractor and cavity inventory, and dissecting the cystic artery and cystic duct.

The cystic duct will be opened and its catheterization and aspiration of $1 \mathrm{ml}$ of bile will be carried out, which will be sent for analysis of the microbiota.

Subsequently, intraoperative cholangiography will be performed to study the intra and extrahepatic biliary anatomy, followed by anterograde cysticfunicular cholecystectomy and left or right hepatectomy as clinical indication.

\section{- Processing of samples}

Sample collections will be sent to the Molecular Biology Sector of the Central Laboratory Division (LIM-03), which will be responsible for DNA extraction, construction of $16 \mathrm{~S}$ libraries and sequencing of microbiomes.

- DNA Extraction

DNA extraction will be performed from $1 \mathrm{ml}$ of the content of the e-swab, using the DNA Kit Zymobiomics MiniPrep, according to the manufacturer's recommendations. The DNA will be stored immediately at $-20^{\circ} \mathrm{C}$ until use.

\section{- Sequencing of microbiomes}


The sequencing of microbiomes will be performed using the 16S Metagenomic Sequencing Library protocol (Illumina, San Diego, USA). In parallel, the microbiomes will also be sequenced based on total DNA extracted from the samples, for a more complete assessment of the bacterial species found in each patient. Genomic DNA libraries will be built using Nextera ${ }^{\circledR}$ XT DNA Sample Preparation Kit (Illumina) from an input of $1 \mu \mathrm{g}$ of DNA, according to the manufacturer's recommendations. The $16 \mathrm{~S}$ and genomic DNA libraries will be sequenced in MiSeq (Illumina, San Diego, USA), using the MiSeq ${ }^{\circledR}$ Reagent Kit v3 (600 cycles; Illumina, San Diego, USA).

\section{- Data analysis}

The data analyses will be performed at the Bioinformatics Laboratory (Setulab), located in the Biochemistry Department, at the Chemistry Institute of the University of São Paulo.

Sequencing the $16 \mathrm{~S}$ amplicons (bacteria) from the different samples will result in sets of reads. The data will be sent to Setulab servers, where the analyses will be carried out, which will mainly follow the steps and programs available in the QIIME 2 package [14]. Briefly, the steps include quality control, to remove short reads or medium grades below a minimum limit; determination of amplicon sequence variants (ASVs) using the deblur [15] and / or DADA2 [16] programs; taxonomic classification of ASVs; alpha and beta diversity analyses; rarefaction analysis; and several statistical analyses, seeking to show differences in the microbial composition between control and patient samples. In the latter case, in addition to the QIIME 2 packages, we will use the STAMP program [17]. The results will be made available mainly in the form of tables and graphs.

The analysis of total DNA data will follow the following steps:

1. Quality control for the removal of short reads or average quality below a threshold.

2. Quality control to separate DNA from the microbiota from any human DNA that has been sequenced.

3. Classification of reads through the centrifuge program [18].

4. Application of the metaWrap pipeline [19] to recover data genomes (generation of MAGs). The application will be separated by sample, and the results will be compared later.

5. Classification of MAGs using the GTDB-tk program [20].

6. Comparison of the results of taxonomic classification of total DNA data with those obtained by $16 \mathrm{~S}$.

The results obtained will also be compared with results from the literature, in particular through the eHOMD website [21].

- Data management

Once this study is completed, the biological material will receive a code and will be filed in the biorepository anonymously, and may be used for other academic studies, without commercial purpose, as long as approved by the Research Ethics Committee, in accordance with the guidelines of the national body that coordinates the principles of research in our country, the National Commission for Ethics in Research.

- Safety and risks regarding adverse events

Bile collection, both in the case group and in the control group, carries minimal risk and discomfort. However, serious adverse events related to examination (ERCP) and / or hepatectomy surgery will be documented on a form throughout the course of the study and will be reported to the principal investigator within 24 hours of observation. If the event is considered to be related to the collection of bile by the principal investigator, he will send a report to the local ethics committee within 3 days.

\section{- Timetable}

The research is estimated to last twenty-four months, according to the following schedule (Table 1)

Table 1

Research schedule

\begin{tabular}{|c|c|c|c|c|c|c|c|c|c|c|c|c|c|c|c|c|c|c|c|c|c|c|c|c|}
\hline Month & 1 & 2 & 3 & 4 & 5 & 6 & 7 & 8 & 9 & 10 & 11 & 12 & 13 & 14 & 15 & 16 & 17 & 18 & 19 & 20 & 21 & 22 & 23 & 24 \\
\hline $\begin{array}{l}\text { Bibliographic } \\
\text { Survey }\end{array}$ & $x$ & $x$ & $X$ & $x$ & $x$ & $x$ & & & & & & & & & & & & & & & & & & \\
\hline $\begin{array}{l}\text { Sample } \\
\text { collection }\end{array}$ & & & & $x$ & $x$ & $x$ & $x$ & $x$ & $x$ & $x$ & $x$ & $x$ & $x$ & $x$ & $x$ & $x$ & $x$ & & & & & & & \\
\hline $\begin{array}{l}\text { Sample } \\
\text { processing }\end{array}$ & & & & & & & & $x$ & $x$ & $x$ & $x$ & $x$ & $x$ & $x$ & $x$ & $x$ & $x$ & & & & & & & \\
\hline $\begin{array}{l}\text { Data } \\
\text { tabulation }\end{array}$ & & & & & & & & & & & & & & & $x$ & $x$ & $x$ & $x$ & & & & & & \\
\hline $\begin{array}{l}\text { Results } \\
\text { analysis }\end{array}$ & & & & & & & & & & & & & & & & & & $x$ & $x$ & $x$ & $x$ & $x$ & & \\
\hline $\begin{array}{l}\text { Completion } \\
\text { of work }\end{array}$ & & & & & & & & & & & & & & & & & & & & & & & $x$ & $x$ \\
\hline
\end{tabular}




\section{Statistical analysis}

All data will be presented as average and standard deviation. Student's t-test will be performed with SPSS version 20 for Windows and the MannWhitney test will be performed using R software and Python scripts. These tests will be used for comparison, as appropriate. A PCA signals will be developed as discriminative analysis.

The sample size calculation, according to the population under investigation, was estimated at 40, with 20 from each group, with an effect size of 0.98 , type I error of $5 \%$ and power of $80 \%[23,26]$.

\section{Result Parameters}

The primary purpose of the study is to characterize the specific composition of the biliary microbiota in situations of disease and health. For this, the microbiota of patients with hepatobiliopancreatic diseases will be compared with that of healthy controls. In addition, it seeks to demonstrate the existence of changes in the case of illness.

Further investigation includes disease biomarkers, diagnosis, interventions and therapies in hepatobiliopancreatic diseases.

\section{Dissemination policy}

MICROBILIO results should be presented at international medical conferences on corresponding areas of interest, for example, gastroenterology. Written publications will be submitted to surgical or endoscopic scientific journals. The authorship of written publications must be confirmed unequivocally by all leading researchers.

\section{Protocol version}

This manuscript refers to the first version of the complete study protocol, made on June 4th, 2020. Modifications to the protocol will be reported to all investigators, the local Research Ethics Committee, all participants and the journal.

\section{Discussion}

The composition and role of the microbiota of the gastrointestinal tract is an increasing focus of study. There is even evidence that these microbes are related to cancer and other chronic diseases [5]. Biliary dysbiosis, for example, has been shown to be a protagonist in the pathogenesis of several diseases in this tract, as has been recently described. However, given the proximity and connection between the pancreatic, hepatic and biliary tracts, it may be that the influence of this microbiota is more extensive, reaching the entire hepatobiliopancreatic tract [22].

On the other hand, the bile microbiota is still little studied, being sequenced in healthy individuals only recently [23]. Little is known about its composition in malignant diseases [24], with biliary lithiasis being the most studied and best characterized disease [23, 25].

This study aims to consolidate existing knowledge and deepen it with additional information. Thus, we seek to broaden the panorama about the different compositions of the biliary microbiota and, possibly, to shed light on a new form of study for hepatobiliopancreatic diseases, as well as strategies on how to deal with such conditions.

\section{Conclusion}

We expect that this study will provide new information about the biliary tract microbiome in both disease and health conditions. We also expect to make progress in the development of biomarkers, diagnosis, interventions and therapies to hepatobiliopancreatic diseases. Thus, we believe that the investigation of the biliary tract microbiota in this study will provide improvements in quality of life and survival, specially in malignant diseases.

\section{Declarations}

\section{Ethics approval and consent to participate}

The study protocol was approved by the Hospital Ethics Committee (Faculty of Medicine, University of São Paulo - FMUSP - CAAE:

29547920.9.0000.0068). The study was registered at ClincalTrials.gov (Identifier: NCT04391426).

\section{Consent for publication}

Not applicable. 


\section{Availability of data and material}

All data generated or analysed during this study are included in this published article and its supplementary information files.

\section{Competing interests}

The authors declare that they have no competing interests

\section{Funding}

No funding.

\section{Authors' contributions}

FSN and MOS: Conception, methodology, formal analysis, investigation, writing, drafted the work; JVT, VCM, LZP and EMCD: Validation, review; JRRP, WA, EGHM and LACD: Supervision, project administration; AM: Conception, methodology, investigation, supervision, project administration; JCS: Methodology, investigation, processing of samples and analysis.

All authors have approved the submitted version and have agreed both to be personally accountable for the author's own contributions and to ensure that questions related to the accuracy or integrity of any part of the work, even ones in which the author was not personally involved, are appropriately investigated, resolved, and the resolution documented in the literature.

\section{Acknowledgements}

The authors are thankful to Justin Axel-Berg for the English corrections and Rossana V. Mendoza López for the statistical analysis.

\section{Research Status}

This project is suspended due to the COVID-19 pandemic, which makes it impossible to recruit and collect data, scheduled to resume in August 2020.

\section{References}

1. Sender R, Fuchs S, Milo R. Revised Estimates for the Number of Human and Bacteria Cells in the Body. PLoS Biol. 2016;14(8):e1002533.

2. Cresci GA, Bawden E. Gut Microbiome: What We Do and Don't Know. Nutr Clin Pract. 2015;30(6):734-746.

3. Akshintala VS, Talukdar R, Singh VK, Goggins M. The Gut Microbiome in Pancreatic Disease. Clin Gastroenterol Hepatol. 2019;17(2):290-295.

4. Mitsuhashi K, Nosho K, Sukawa Y, et al. Association of Fusobacterium species in pancreatic cancer tissues with molecular features and prognosis. Oncotarget. 2015;6(9):7209-7220.

5. Saus E, Iraola-Guzmán S, Willis JR, Brunet-Vega A, Gabaldón T. Microbiome and colorectal cancer: Roles in carcinogenesis and clinical potential. Mol Aspects Med. 2019;69:93-106.

6. Riquelme E, Zhang Y, Zhang L, et al. Tumor Microbiome Diversity and Composition Influence Pancreatic Cancer Outcomes. Cell. 2019;178(4):795806.e12.

7. Aykut B, Pushalkar S, Chen R, et al. The fungal mycobiome promotes pancreatic oncogenesis via activation of MBL. Nature. 2019;574(7777):264267.

8. Mendez R, Kesh K, Arora N, et al. Microbial dysbiosis and polyamine metabolism as predictive markers for early detection of pancreatic cancer [published online ahead of print, 2019 Aug 1]. Carcinogenesis. 2019;bgz116.

9. Mima K, Nakagawa S, Sawayama H, et al. The microbiome and hepatobiliary-pancreatic cancers. Cancer Lett. 2017;402:9-15.

10. lida N, Dzutsev A, Stewart CA, et al. Commensal bacteria control cancer response to therapy by modulating the tumor microenvironment. Science. 2013;342(6161):967-970.

11. Wong SH, Yu J. Gut microbiota in colorectal cancer: mechanisms of action and clinical applications. Nat Rev Gastroenterol Hepatol. 2019;16(11):690-704.

12. Desrouillères $K$, Millette $M$, Bagheri L, Maherani B, Jamshidian M, Lacroix M. The synergistic effect of cell wall extracted from probiotic biomass containing Lactobacillus acidophilus CL1285, L. casei LBC80R, and L. rhamnosus CLR2 on the anticancer activity of cranberry juice-HPLC fractions. J Food Biochem. 2020;44(5):e13195. 
13. Lenoir M, Del Carmen S, Cortes-Perez NG, et al. Lactobacillus casei BL23 regulates Treg and Th17 T-cell populations and reduces DMH-associated colorectal cancer. J Gastroenterol. 2016;51(9):862-873.

14. Bolyen E, Rideout JR, Dillon MR, et al. Reproducible, interactive, scalable and extensible microbiome data science using QIIME 2 [published correction appears in Nat Biotechnol. 2019 Sep;37(9):1091]. Nat Biotechnol. 2019;37(8):852-857.

15. Amir A, McDonald D, Navas-Molina JA, et al. Deblur Rapidly Resolves Single-Nucleotide Community Sequence Patterns. mSystems. 2017;2(2):e00191-16.

16. Callahan BJ, McMurdie PJ, Rosen MJ, Han AW, Johnson AJ, Holmes SP. DADA2: High-resolution sample inference from Illumina amplicon data. Nat Methods. 2016;13(7):581-583.

17. Parks DH, Tyson GW, Hugenholtz P, Beiko RG. STAMP: statistical analysis of taxonomic and functional profiles. Bioinformatics. 2014;30(21):3123-3124.

18. Kim D, Song L, Breitwieser FP, Salzberg SL. Centrifuge: rapid and sensitive classification of metagenomic sequences. Genome Res. 2016;26(12):1721-1729.

19. Uritskiy GV, DiRuggiero J, Taylor J. MetaWRAP-a flexible pipeline for genome-resolved metagenomic data analysis. Microbiome. $2018 ; 6$ (1):158.

20. Parks DH, Chuvochina M, Waite DW, et al. A standardized bacterial taxonomy based on genome phylogeny substantially revises the tree of life. Nat Biotechnol. 2018;36(10):996-1004.

21. Escapa IF, Chen T, Huang Y, Gajare P, Dewhirst FE, Lemon KP. New Insights into Human Nostril Microbiome from the Expanded Human Oral Microbiome Database (eHOMD): a Resource for the Microbiome of the Human Aerodigestive Tract. mSystems. 2018;3(6):e00187-18.

22. Nicoletti A, Ponziani FR, Nardella E, laniro G, Gasbarrini A, Zileri Dal Verme L. Biliary tract microbiota: a new kid on the block of liver diseases?. Eur Rev Med Pharmacol Sci. 2020;24(5):2750-2775.

23. Molinero N, Ruiz L, Milani C, et al. The human gallbladder microbiome is related to the physiological state and the biliary metabolic profile. Microbiome. 2019;7(1):100.

24. Chen B, Fu SW, Lu L, Zhao H. A Preliminary Study of Biliary Microbiota in Patients with Bile Duct Stones or Distal Cholangiocarcinoma. Biomed Res Int. 2019;2019:1092563.

25. Shen H, Ye F, Xie L, et al. Metagenomic sequencing of bile from gallstone patients to identify different microbial community patterns and novel biliary bacteria. Sci Rep. 2015;5:17450.

26. Gao Z, Guo B, Gao R, Zhu Q, Qin H. Microbiota disbiosis is associated with colorectal cancer. Front Microbiol. $2015 ; 6: 20$.

\section{Figures}




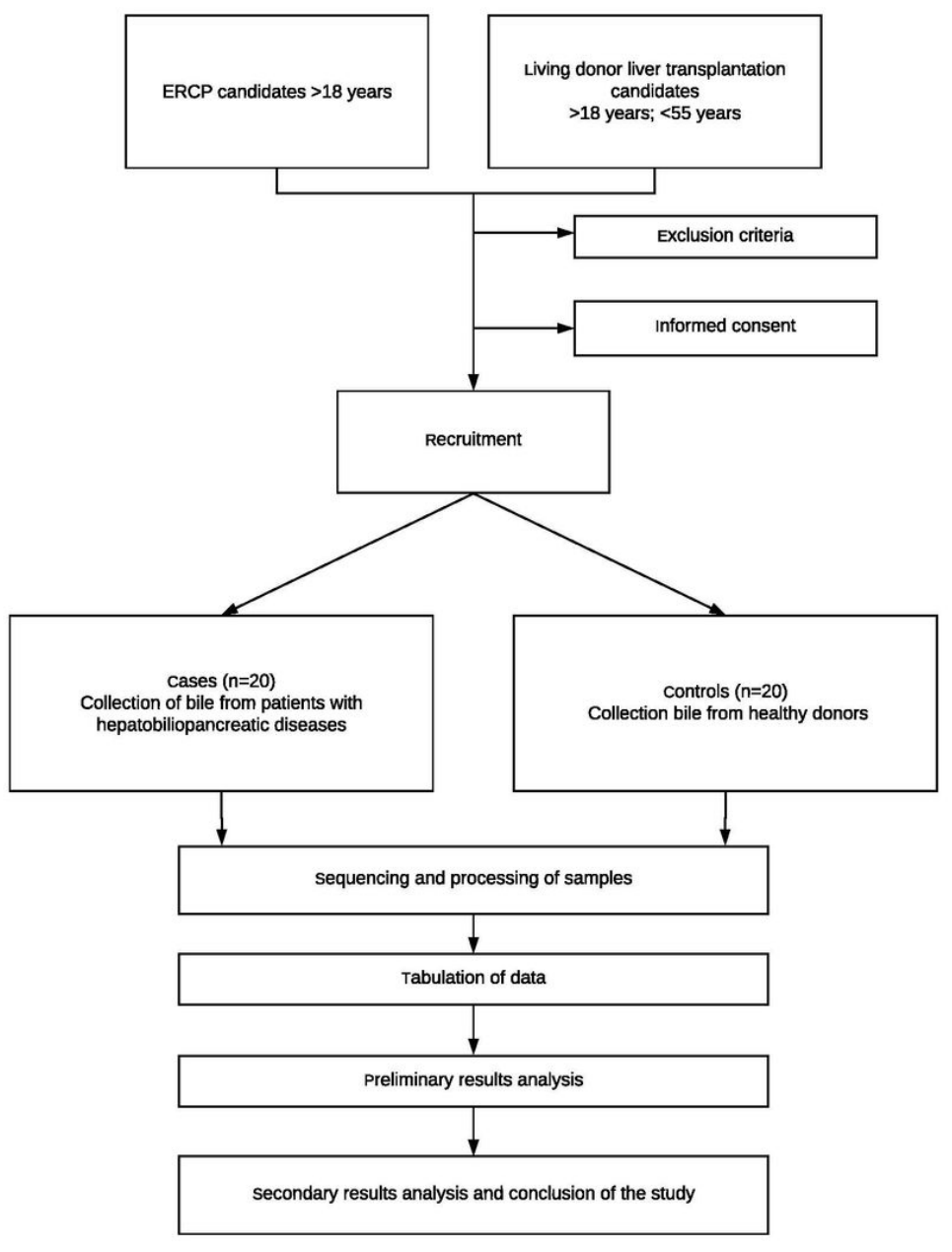

Figure 1

Study scheme 